\title{
Do biomarkers have predictive value in the treatment modality of the patients diagnosed with bowel obstruction?
}

\author{
Gonca Koksaldi Sahin ${ }^{1 *}$ (D), Muge Gulen ${ }^{1}$ (D), Selen Acehan ${ }^{1}$ (D), Basak Toptas Firat ${ }^{1}$ (D), Cem Isikber ${ }^{1}$ (D), \\ Adem Kaya ${ }^{1}$ (D), Mustafa Sencer Segmen ${ }^{1}$ (D), Yeliz Simsek ${ }^{1}$ (D), Alper Sozutek ${ }^{2}$ (D), Salim Satar ${ }^{1}$ (i)
}

\begin{abstract}
SUMMARY
OBJECTIVES: This study aimed to investigate the ability of the biomarkers to predict the surgery treatment and mortality in patients above 18 years of age who were hospitalized with the diagnosis of bowel obstruction from the emergency department.

METHODS: This is a 2-year retrospective study. The patients' demographic data, laboratory parameters on admission to emergency department, treatment modalities, and the length of hospital stay were recorded. Patients were divided into two groups: conservative and surgical treatment. Statistical analysis was performed to investigate the value of biomarkers in predicting mortality and the need for surgery. Data were analyzed using IBM SPSS version 22.

RESULTS: A total of 179 patients were included in this study. Of these, 105 (58.7\%) patients were treated conservative and 74 (41.3\%) were treated operatively. The elevated procalcitonin (PCT) level, C-reactive protein, blood urea nitrogen-to-albumin ratio, and lactate-to-albumin ratio were significantly correlated with surgical treatment, length of hospital stay, and mortality. procalcitonin threshold value of $0.13 \mathrm{ng} / \mathrm{mL}$ was able to predict the need for surgical treatment, with a sensitivity of $79 \%$ and a specificity of $70.3 \%$. Procalcitonin threshold value of $0.65 \mathrm{ng} / \mathrm{mL}$ was able to predict the mortality rate of the patients, with a sensitivity of $92.9 \%$ and a specificity of $78.1 \%$.

CONCLUSIONS: Biomarkers, especially procalcitonin, may be useful in bowel obstruction treatment management and may predict mortality. KEYWORDS: Intestinal obstruction. Emergency. Mortality. Procalcitonin. Surgical procedures, operative.
\end{abstract}

\section{INTRODUCTION}

The rapid increase in the population has led to a large increase in the number of patients admitted to emergency services. Approximately $5-10 \%$ of emergency department admissions are due to bowel obstruction $^{1}$. There are two approaches to the treatment of bowel obstruction: conservative and surgical. Patients who fail to improve within 48-72 h with conservative treatment need surgical treatment. In this process, bowel ischemia and necrosis may progress ${ }^{2,3}$.

Procalcitonin (PCT) is released from the $\mathrm{C}$ cells of the thyroid in healthy individuals. It has been established that the source of increased PCT after bacterial infections is the neuroendocrine cells in the lungs, liver, intestines, and pancreas ${ }^{4-6}$. The half-life of PCT ranges from 18 and $24 \mathrm{~h}^{7}$. Plasma levels $>0.5 \mathrm{ng} / \mathrm{mL}$ are considered pathological ${ }^{8,9}$.

C-reactive protein (CRP) was the first acute-phase reactant detected to be susceptible to inflammation and tissue damage. An increased lactate level is an important marker for indicating tissue hypoxia, sepsis, and mortality. Studies show that the CRP/lactate ratio predicts mortality in intestinal obstruction ${ }^{3,10}$. Preoperative low albumin levels are related to extracellular interstitial fluid and may predict mortality ${ }^{11,12}$. Blood urea nitrogen $(\mathrm{BUN}) /$ albumin ratio can predict mortality in critically ill patients ${ }^{13}$.

In this study, we aimed to investigate the ability of laboratory values (i.e., PCT, CRP/lactate, and BUN/albumin) to predict the need for surgical treatment and mortality in patients diagnosed with bowel obstruction.

\section{METHODS}

This study, which was planned retrospectively, included patients who were diagnosed as having ileus in the emergency department of a tertiary care hospital and hospitalized from January 1 , 2018 to December 31, 2020. This study obtained the approval from the ethics committee.

\section{Patients}

Patients diagnosed with ileus (diagnosis was made by emergency physicians) in the emergency department were included in this

\footnotetext{
${ }^{1}$ Adana City Training and Research Hospital, Department of Emergency Medicine - Adana, Turkey.

${ }^{2}$ Adana City Training and Research Hospital, Department of General Surgery - Adana, Turkey.

${ }^{*}$ Corresponding author: epostagks@gmail.com

Conflicts of interest: the authors declare there is no conflicts of interest. Funding: none.

Received on September 15, 2021. Accepted on September 16, 2021.
} 
study. This study consisted of 179 hospitalized patients aged above 18 years with acute abdomen and diagnosed as having ileus. The exclusion criteria were as follows: patients with acute mesenteric ischemia, postoperative early obstruction, cancer diagnoses, patients aged below 18 years, patients who did not accept treatment, those who left the emergency department without permission, and patients referred to another hospital.

\section{Data collection and measurements}

Relevant data were collected from the records of the emergency department and the hospital data processing record system. Data analysis included demographic data, laboratory parameters on admission to the emergency department, computed tomography (CT) reports, treatment modalities (conservative or surgical), length of hospital stay (LOS), and mortality. Serum PCT levels were analyzed using chemiluminescence immunoassay (CLIA), and a standard test kit was used. Other biochemical parameters were studied using a Beckman Coulter Chemistry Analyzer AU5800.

We divided the patients into two groups: those who underwent surgery and those hospitalized for conservative treatment with nasogastric decompression. The decision for surgery or conservative treatment was made after consultation with a general surgeon based on the clinical and radiologic findings, the presence of obstruction, and the presence of strangulation, fluidelectrolyte, and acid-base balance. We compared the PCT, CRP, lactate, $\mathrm{CRP} /$ albumin, lactate/albumin, and BUN/albumin values in these patients to predict surgical treatment and mortality.

\section{Outcome}

The first outcome for this study was in-hospital mortality. The patients were followed up during hospitalization for mortality. The secondary outcomes were the patients' need for surgical treatment and LOS.

\section{Statistical analysis}

Data were analyzed using IBM SPSS version 22. Mean, standard deviation, median, minimum-maximum, frequency, and ratio values were used in the descriptive statistics of the data. The $\chi^{2}$ test was used for the comparison of categorical variables. Student's $t$-test was performed to compare two groups when normally distributed, and Mann-Whitney $U$ test when not normally distributed. A receiver operating characteristics (ROC) curve was used to investigate the accuracy of PCT and other biomarkers in predicting mortality and the need for surgery. The cutoff value was determined using the Youden Index, in which the highest sensitivity and specificity point in the ROC curve was taken. The statistical significance level was set as $\mathrm{p}<0.05$.

\section{RESULTS}

The total number of patients admitted to the emergency department during the study period (January 1, 2018, to December 31,2020 ) was 576,499 . Of these, 18,374 patients were hospitalized in the general surgery clinic from emergency department. Of these, 8,841 patients were hospitalized for nontraumatic reasons. Notably, 378 patients included in the study were those who were diagnosed with ileus (diagnosed by emergency medicine physicians) on admission to the emergency department and were hospitalized in the general surgery clinic with this preliminary diagnosis. Overall, 199 patients were excluded from this study: 155 patients had a diagnosis other than ileus (acute appendicitis $n=17$, acute cholecystitis $n=46$, acute mesenteric ischemia $n=13$, acute diverticulitis $n=7$, strangulated hernia $n=15$, perforated gallbladder $n=5$, four gastric perforation $\mathrm{n}=4$, and tumor $\mathrm{n}=48$ ), 4 patients did not accept hospitalization despite having bowel obstruction, 34 patients had missing file information and laboratory parameters, and 6 patients left the emergency department without permission.

As a result, 179 patients were included in the final analysis; $39.1 \%(\mathrm{n}=70)$ were female and $60.9 \%(\mathrm{n}=109)$ were male. The mean age of the patients was $56.5 \pm 7.3$ years. The distribution, demographic characteristics, and laboratory parameters of the patients according to the treatment they received are presented in Table 1.

The first treatment methods that the patients received after being admitted to the surgery clinic due to ileus were as follows: conservative treatment with nasogastric decompression was performed on $58.7 \%$ of the patients $(n=105)$, and surgical treatment was performed on $41.3 \%(n=74)$. The mean LOS of the patients who received conservative treatment in hospital was 3.6 62.1 days and that of patients who received surgi-

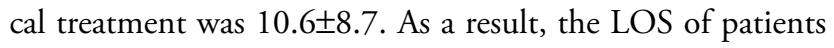
who needed surgical treatment was considerably longer than in patients who received conservative treatment $(\mathrm{p}<0.001)$.

Figure 1 presents the need for surgical treatment of PCT, lactate, CRP/lactate, lactate/albumin, and BUN/albumin values from the laboratory data at the time of hospital admission, as determined using ROC curve analysis. In direct comparisons, PCT showed better overall performance than lactate, CRP/lactate, lactate/albumin, and BUN/albumin, with the area under the ROC curve (AUC) value of 0.798 (95\%CI $0.730-0.866$ ). A PCT threshold value of $0.13 \mathrm{ng} / \mathrm{mL}$ was able to predict the need for surgical treatment, with a sensitivity of $79 \%$ and specificity of $70.3 \%$

Finally, 137 (76.5\%) patients were discharged, and 24.5\% $(n=42)$ died. ROC analysis was also performed to predict the mortality rate of the patients using PCT, lactate, CRP/lactate, 
lactate/albumin, and BUN/albumin values from the laboratory data at the time of hospital admission, as shown in Figure 1. The analysis of this curve is presented in Table 2. PCT showed better results than the other parameters, with an AUC value of 0.921 (95\%CI 0.882-0.960; $\mathrm{p}<0.001)$. A PCT threshold value of $0.65 \mathrm{ng} / \mathrm{mL}$ was able to predict mortality in patients, with a sensitivity of $92.9 \%$ and a specificity of $78.1 \%$.
In the analyses for the prediction of mortality, the lactate/ albumin ratio (AUC 0.788, 95\%CI 0.705-0.872; p<0.001) was higher than the BUN/albumin value (AUC 0.715, 95\%CI $0.629-0.802 ; \mathrm{p}<0.001)$. The lactate/albumin threshold value of 0.075 was able to predict mortality with a sensitivity of $78.6 \%$ and a specificity of $63.5 \%$, and the BUN/albumin threshold value was 0.69 with a sensitivity of $88.1 \%$ and specificity of $54.7 \%$.

Table 1. Characteristics of the patients according to the requirement of treatment.

\begin{tabular}{|c|c|c|c|}
\hline & $\begin{array}{c}\text { Conservative } \\
\text { management } \\
(n=74)\end{array}$ & $\begin{array}{c}\text { Surgical } \\
\text { management } \\
(n=105)\end{array}$ & p-value \\
\hline Age, years (min-max) & $53.4 \pm 6.3(65-87)$ & $57.7 \pm 8.3(65-97)$ & 0.034 \\
\hline \multicolumn{4}{|l|}{ Sex, n (\%) } \\
\hline Female & $37(50)$ & $33(31.4)$ & \multirow{2}{*}{0.012} \\
\hline Male & $37(50)$ & $72(68.6)$ & \\
\hline \multicolumn{4}{|l|}{ Comorbidity, n (\%) } \\
\hline Hypertension & $36(48.6)$ & $67(63.8)$ & 0.043 \\
\hline Coronary artery disease & $23(31.1)$ & $52(49.5)$ & 0.014 \\
\hline Lung disease & $19(25.7)$ & $30(28.6)$ & 0.669 \\
\hline Kidney insufficiency & $11(14.9)$ & $37(35.2)$ & 0.002 \\
\hline Diabetes mellitus & $16(21.6)$ & $23(21.9)$ & 0.964 \\
\hline Congestive heart failure & $4(5.4)$ & $17(16.2)$ & 0.027 \\
\hline Cerebrovascular disease & $10(13.5)$ & $8(7.6)$ & 0.197 \\
\hline BT Finding & $64(86.5)$ & $94(89.5)$ & 0.534 \\
\hline Length of hospital stay (days) & $3.6 \pm 2.1$ & $10.6 \pm 8.7$ & $<0.001$ \\
\hline \multicolumn{4}{|l|}{ Prognosis } \\
\hline Survival & $69(93.2)$ & $68(64.8)$ & \multirow{2}{*}{$<0.001$} \\
\hline Non-survival & $5(6.8)$ & $37(35.2)$ & \\
\hline White blood cell $\left(10^{3} / \mu \mathrm{L}\right)$ & $12.1 \pm 5.8$ & $12.1 \pm 6.1$ & 0.938 \\
\hline Hemoglobin (g/dL) & $12.7 \pm 2.1$ & $12.5 \pm 2.5$ & 0.549 \\
\hline Platelet $\left(10^{3} / \mu \mathrm{L}\right)$ & $314.7 \pm 140.9$ & $292.9 \pm 127.1$ & 0.281 \\
\hline \multicolumn{4}{|l|}{ Biochemical Parameters } \\
\hline Sodium (mmoL/L) & $136.8 \pm 4.7$ & $135.7 \pm 4.9$ & 0.171 \\
\hline Potassium (mmoL/L) & $4.5 \pm 0.6$ & $4.3 \pm 0.8$ & 0.014 \\
\hline Chlorine (mmoL/L) & $100.9 \pm 5$ & $99.6 \pm 6.3$ & 0.140 \\
\hline Calcium (mmoL/L) & $9.4 \pm 0.8$ & $8.9 \pm 0.9$ & 0.002 \\
\hline Blood urea nitrogen (mg/dL) & $26.1 \pm 14.8$ & $32.0 \pm 17.7$ & 0.015 \\
\hline Creatinine (mg/dL) & $1.05 \pm 0.7$ & $1.6 \pm 2.2$ & 0.026 \\
\hline Aspartate transaminase (U/L) & $25.1 \pm 12.2$ & $37.2 \pm 55.5$ & 0.069 \\
\hline Alanine transaminase (U/L) & $16.8 \pm 10$ & $24.1 \pm 33.2$ & 0.067 \\
\hline Albumin (mg/L) & $35.6 \pm 5.8$ & $31.2 \pm 7.6$ & $<0.001$ \\
\hline C-reactive protein (mg/L) & $8.5 \pm 9.8$ & $15.4 \pm 13.5$ & $<0.001$ \\
\hline Procalcitonin (ng/mL) & $2.2 \pm 11.1$ & $7.5 \pm 15.8$ & 0.009 \\
\hline Lactate (mmoL/L) & $2.2 \pm 1.1$ & $3.2 \pm 2.5$ & $<0.001$ \\
\hline Lactate/albumin & $0.06 \pm 0.03$ & $0.11 \pm 0.08$ & $<0.001$ \\
\hline BUN/albumin & $0.8 \pm 0.5$ & $1.1 \pm 0.7$ & $<0.001$ \\
\hline
\end{tabular}

BUN: blood urea nitrogen. 


\section{DISCUSSION}

Our study investigated the ability to predict surgery and mortality using laboratory parameters checked at the time of hospital admission in patients who were hospitalized due to ileus from the emergency department. We observed that high PCT values in patients with ileus were greater than other laboratory markers compared to predicting the need for surgical treatment and mortality. We found that the need for surgical treatment increased with PCT values $>0.13 \mathrm{ng} / \mathrm{mL}$ (sensitivity $79 \%$, specificity $70.3 \%$ ) and mortality significantly increased at values $>0.65 \mathrm{ng} / \mathrm{mL}$ (sensitivity $92.9 \%$, specificity $78.1 \%$ ).
Laboratory parameters affected by inflammatory markers are also expected to increase. These mediators induce the release of PCT from intestinal macrophages and hepatocytes through the portal system ${ }^{14}$. A prospective non-randomized study conducted with 59 patients diagnosed with small bowel obstruction showed that the surgical treatment achieved cutoff value by measuring PCT levels every $6 \mathrm{~h}$. The cutoff value was $0.16 \mathrm{ng} / \mathrm{mL}$ at first admission and was $>0.27 \mathrm{ng} / \mathrm{mL}$ at the 18 th hour ${ }^{15}$. In another study of 242 cases that predicted surgical treatment, the authors emphasized that PCT levels in the treatment of ileus could play an essential role in clinical

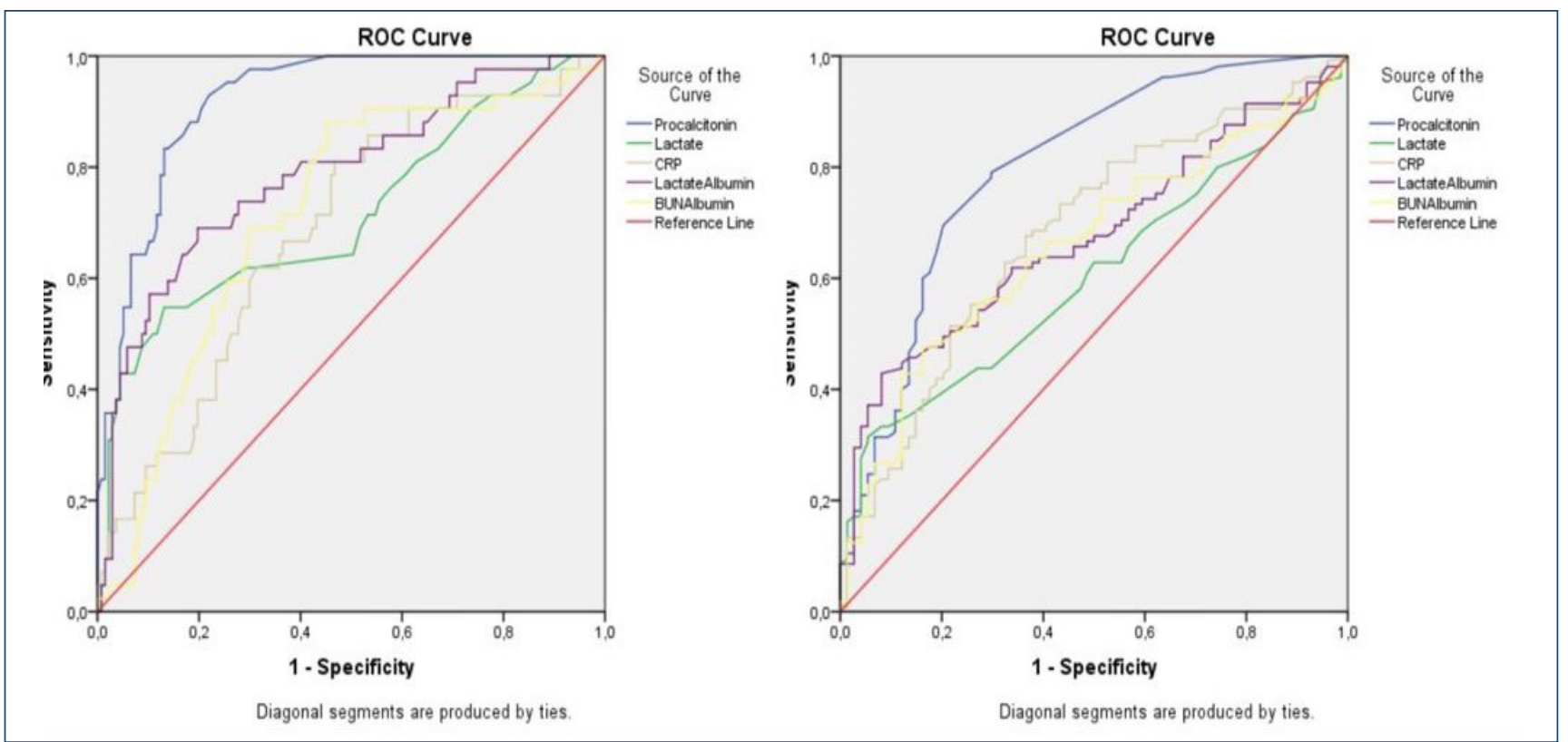

Figure 1. Receiver operating characteristics curves showing comparisons of procalcitonin, lactate, C-reactive protein, lactate/albumin, blood urea nitrogen/albumin values in predicting $(A)$ the need for surgery and $(B)$ hospital mortality.

Table 2. Receiver operating characteristics analysis of procalcitonin, lactate, C-reactive protein, lactate/albumin, and blood urea nitrogen/albumin values for the need of surgery and hospital mortality.

AUC $\quad$ SE

$(95 \% \mathrm{Cl})$

Cutoff

Sensitivity $\quad$ Specificity

p-value

Surgery need

\begin{tabular}{l|c|c|c|c|c|c|c}
\hline PCT & 0.798 & 0.035 & $0.730-0.866$ & 0.13 & 79 & 70.3 & $<0.001$ \\
\hline CRP & 0.681 & 0.040 & $0.602-0.760$ & 5.9 & 73.3 & 56.8 & $<0.001$ \\
\hline Lactate/albumin & 0.673 & 0.040 & $0.595-0.751$ & 0.07 & 63.8 & 60.8 & $<0.001$ \\
\hline BUN/albumin & 0.657 & 0.041 & $0.577-0.737$ & 0.73 & 62.9 & 63.5 & $<0.001$ \\
\hline CRP/lactate & 0.615 & 0.043 & $0.532-0.699$ & 2.3 & 69.5 & 51.4 & 0.009 \\
\hline Lactate & 0.603 & 0.042 & $0.522-0.685$ & 2.35 & 62.9 & 50 & 0.019 \\
\hline
\end{tabular}

Hospital mortality

\begin{tabular}{l|c|c|c|c|c|c|c}
\hline PCT & 0.921 & 0.020 & $0.882-0.960$ & 0.65 & 92.9 & 78.1 & $<0.001$ \\
\hline Lactate/albumin & 0.788 & 0.042 & $0.705-0.872$ & 0.075 & 78.6 & 63.5 & $<0.001$ \\
\hline BUN/albumin & 0.715 & 0.044 & $0.629-0.802$ & 0.69 & 88.1 & 54.7 & $<0.001$ \\
\hline Lactate & 0.713 & 0.050 & $0.615-0.811$ & 2.75 & 61.9 & 70.8 & $<0.001$ \\
\hline CRP & 0.686 & 0.046 & $0.597-0.775$ & 8 & 81 & 53.3 & $<0.001$ \\
\hline
\end{tabular}

BUN: blood urea nitrogen; CRP: C-reactive protein; PCT: procalcitonin; ROC: receiver operating characteristics. 
decision-making in acute bowel obstruction, and they proceeded to surgery with value $>1 \mathrm{ng} / \mathrm{mL}^{16}$.

Our study found that CRP and lactate were also significant in predicting surgical treatment. CRP begins to rise in 3-6 $\mathrm{h}$ after inflammation, peaks in 30-60 $\mathrm{h}^{17}$. Studies suggested that CRP and lactate levels might help predict strangulation and make early decisions for surgery ${ }^{18}$.

Lactate is produced from many tissues, mostly muscle tissue. It has two isomers, L-lactate and D-lactate. D-Lactate is used by human colon bacteria, produced by glycosylase, and its normal serum level is very low. Its elevation is always associated with pathological bowel diseases and strangulation ${ }^{19}$.

It was reported that BUN levels increased in critical diseases with high mortality ${ }^{13,20,21}$. Albumin, a negative acutephase reactant, is a protein that protects plasma osmolarity. The decrease in albumin synthesis due to nutrition and inflammation causes a decrease in serum albumin level ${ }^{22}$. Our study observed that the ratio of BUN and albumin in patients with intestinal obstruction aged above 18 years was significant in determining LOS and mortality. Especially in patients with impaired intestinal obstruction, increased BUN and decreased albumin values may have an essential place in predicting mortality.

A few studies on PCT and its ability to predict mortality have found different results. In a prospective study of 153 patients who underwent abdominal surgery, no statistically significant relationship was found between PCT and 28-day mortality after surgical treatment ${ }^{23}$, but PCT values were associated with 28 -day mortality $>2.5 \mathrm{ng} / \mathrm{mL}$ in 454 patients who underwent surgical treatment in another study ${ }^{24}$. Our study

\section{REFERENCES}

1. Stefanidis D, Richardson WS, Chang L, Earle DB, Fanelli RD. The role of diagnostic laparoscopy for acute abdominal conditions: an evidence-based review. Surg Endosc. 2009;23(1):16-23. https:// doi.org/10.1007/s00464-008-0103-x

2. Luzny J, Valihrachova S, Novak J, Zapletal T, Donek E, Arogunmati O. Ileus states - conditions connecting psychiatrists and surgeons? Biomed Pap Med Fac Univ Palacky Olomouc Czech Repub. 2010;154(3):261-4. https://doi.org/10.5507/bp.2010.040

3. Markogiannakis H, Messaris E, Dardamanis D, Pararas N, Tzertzemelis D, Giannopoulos P, et al. Acute mechanical bowel obstruction: clinical presentation, etiology, managementand outcome. World J Gastroenterol. 2007;13(3):432-7. https://doi.org/10.3748/wjg.v13.i3.432

4. Cosse C, Regimbeau JM, Fuks D, Mauvais F, Scotte M. Serum procalcitonin for predicting the failure of conservative management and the need for bowel resection in patients with small bowel obstruction. J Am Coll Surg. 2013;216(5):997-1004. https://doi. org/10.1016/j.jamcollsurg.2012.12.051 found that PCT value $>0.65 \mathrm{ng} / \mathrm{mL}$ had a high predictive power for mortality.

The limitations of this study are that it is single-centered and retrospectively arranged study.

\section{CONCLUSION}

It is vital to detect intestinal obstruction for emergency surgical decisions and predict whether it will transform into ischemia. PCT, which is measured in the emergency department, can help the surgeon predict mortality as an additional test to physical examinations and radiology in making methods of treatment for patients with intestinal obstruction.

\section{AUTHORS' CONTRIBUTIONS}

GKS: Conceptualization, Data curation, Formal Analysis, Writing - original draft, Writing - review \& editing. MG: Conceptualization, Data curation, Formal Analysis, Writing original draft, Writing - review \& editing. SA: Conceptualization, Formal Analysis, Writing - original draft, Writing - review \& editing. BTF: Formal Analysis, Writing - original draft. AK: Conceptualization, Data curation, Writing - original draft. CI: Conceptualization, Formal Analysis, Writing - original draft, Writing - review \& editing. MSS: Conceptualization, Writing - original draft, Writing - review \& editing. YS: Conceptualization, Writing - original draft, Writing - review \& editing. AS: Conceptualization, Data curation, Writing review \& editing. SS: Conceptualization, Data curation, Formal Analysis, Writing - original draft.

5. Maruna P, Nedelníková K, Gürlich R. Physiology and genetics of procalcitonin. Physiol Res. 2000;49(Suppl 1):S57-61. PMID: 10984072

6. Cossé C, Sabbagh C, Carroni V, Galmiche A, Rebibo L, Regimbeau JM. Impact of a procalcitonin-based algorithm on the management of adhesion-related small bowel obstruction. J Visc Surg. 2017;154(4):231-7. https://doi.org/10.1016/j. jviscsurg.2017.01.004

7. Maisner M. Procalcitonin-a new, innovative infection parameter biochemical and clinical aspects. 3thed. New York: Thieme, Stuttgart; 2000. p.7-14.

8. Meisner M, Tschaikowsky K, Hutzler A, Schick C, Schüttler J. Postoperative plasma concentrations of procalcitonin after different types of surgery. Intensive Care Med. 1998;24(7):680-4. https:// doi.org/10.1007/s001340050644

9. Meisner M, Schmidt J, Hüttner H, Tschaikowsky K. The natural elimination rate of procalcitonin in patients with normal and impaired renal function. Intensive Care Med. 2000;26(Suppl 2):S212-6. https://doi.org/10.1007/BF02900740 
10. Pal AK,Ansari MM, Islam N. Combination of serumc-reactive protein and d-lactate: predictors of strangulation in intestinal obstruction. Panam J Trauma Crit Care Emerg Surg. 2016;5(3):134-9. https:// doi.org/10.5005/jp-journals-10030-1156

11. Doweiko JP, Nompleggi DJ. The role of albumin in human physiology and pathophysiology, Part III: Albumin and disease states. JPEN J Parenter Enteral Nutr. 1991;15(4):476-83. https:// doi.org/10.1177/0148607191015004476

12. Liang WQ, Zhang KC, Li H, Cui JX, Xi HQ, Li JY, et al. Preoperative albumin levels predict prolonged postoperative ileus in gastrointestinal surgery. World J Gastroenterol. 2020;26(11):118596. https://doi.org/10.3748/wjg.v26.i11.1185

13. Dundar ZD, Kucukceran K, Ayranci MK. Blood urea nitrogen to albumin ratio is a predictor of in-hospital mortality in older emergency department patients. Am J Emerg Med. 2021;46:34954. https://doi.org/10.1016/j.ajem.2020.10.008

14. Soybel DI, Zinner MJ. Ileus and the macrophage. Ann Surg. 2003;237(3):316-8. https://doi.org/10.1097/00000658200303000-00004

15. Cosse C, Sabbagh C, Rebibo L, Grelpois G, Galmiche A, Regimbeau $\mathrm{JM}$. Kinetics of PCT in the management of small bowel obstruction: a preliminary report. Surgery Curr Res. 2014;4:3. https://doi. org/10.4172/2161-1076.1000184

16. Markogiannakis H, Memos N, Messaris E, Dardamanis D, Larentzakis A, Papanikolaou D, et al. Predictive value of procalcitonin for bowel ischemia and necrosis in bowel obstruction. Surgery. 2011;149(3):394-403. https://doi.org/10.1016/j.surg.2010.08.007

17. Hamm CW, Nef HM, Rolf A, Möllmann H. Calcium and C-reactive protein hot enough to predict the future? J Am Coll Cardiol. 2011;57(13):1465-7.https://doi.org/10.1016/j.jacc.2010.10.044
18. Dhoon R, Kumawat S, Soni M.L., Rohella M, Sharma S, Karosia V, et al. Prospective study of serum C-reactive protein and D-lactate levels for early prediction of strangulation in intestinal obstruction. Int Surg J. 2019;6(7):2510-3. http://doi.org/10.18203/2349-2902. isj20192983

19. Smith SM, Eng RH, Buccini F. Use of D-lactic acid measurements in the diagnosis of bacterial infections. J Infect Dis. 1986;154(4):65864. http://doi.org/10.1093/infdis/154.4.658

20. Ryu S, Oh SK, Cho SU, You Y, Park JS, Min JH, et al. Utility of the blood urea nitrogen to serum albumin ratio as a prognostic factor of mortality in aspiration pneumonia patients. Am J Emerg Med. 2021;43:175-9. http://doi.org/10.1016/j. ajem.2020.02.045

21. Richter B, Sulzgruber P, Koller L, Steininger M, El-Hamid F, Rothgerber DJ, et al. Blood urea nitrogen has additive value beyond estimated glomerular filtration rate for prediction of long-term mortality in patients with acute myocardial infarction. Eur J Intern Med. 2019;59:84-90.http://doi.org/10.1016/j.ejim.2018.07.019

22. Doweiko JP, Nompleggi DJ. The role of albumin in human physiology and pathophysiology, Part III: Albumin and disease states. JPEN J Parenter Enteral Nutr. 1991;15(4):476-83. http:// doi.org/10.1177/0148607191015004476

23. Szakmany T, Molnar Z. Procalcitonin levels do not predict mortality following major abdominal surgery. Can J Anaesth. 2003;50(10):1082-3. http://doi.org/10.1007/BF03018387

24. Fritz HG, Brandes H, Bredle DL, Bitterlich A, Vollandt R, Specht $\mathrm{M}$, et al. Post-operative hypoalbuminaemia and procalcitonin elevation for prediction of outcome in cardiopulmonary bypass surgery. Acta Anaesthesiol Scand. 2003;47(10):1276-83. http:// doi.org/10.1046/j.1399-6576.2003.00239.x 